INPLASY

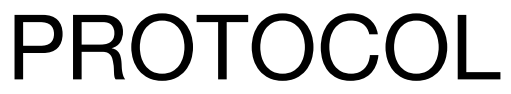

To cite: Wang et al. Effect of electronic health interventions on disease activity, medication compliance and quality of life in rheumatoid arthritis: A metaanalysis. Inplasy protocol 202110069. doi:

10.37766/inplasy2021.1.0069

Received: 18 January 2021

Published: 18 January 2021

Corresponding author: Weiqun Weng

weng1463178375@163.com

Author Affiliation:

Department of Rheumatology and Immunology, the Second Affiliated Hospital of Nantong University

Support: Nantong Municipal Health Commi.

Review Stage at time of this submission: Preliminary searches.

Conflicts of interest:

None.

\section{Effect of electronic health interventions on disease activity, medication compliance and quality of life in rheumatoid arthritis: A meta-analysis}

Wang, J1; Yang, PY2; Cao, HL3; Ben, JL4; Gao, YY5; Wang, XQ6; Weng, WQ7.

Review question / Objective: The review aimed to evaluate the effect of electronic health interventions on disease activity, medication compliance and quality of life in rheumatoid arthritis. A systematic review and meta-analysis of randomized controlled trials.

Condition being studied: Rheumatoid arthritis.

Information sources: We researched PubMed, Cumulative Index Nursing and Allied Health Literature, Cochrane Central Register of Controlled Trials, Web of Science and Embase up to December 2020. In addition, we also conducted manual searches of potentially eligible references.

INPLASY registration number: This protocol was registered with the International Platform of Registered Systematic Review and Meta-Analysis Protocols (INPLASY) on 18 January 2021 and was last updated on 18 January 2021 (registration number INPLASY202110069).

\title{
INTRODUCTION
}

Review question / Objective: The review aimed to evaluate the effect of electronic health interventions on disease activity, medication compliance and quality of life in rheumatoid arthritis. A systematic review and meta-analysis of randomized controlled trials.
Rationale: Rheumatoid arthritis (RA) is a chronic inflammatory rheumatic disease that may lead to disability and impaired health related quality of life. Although RA is incurable, disease-modifying antirheumatic drugs (DMARDs), and biologic agents can control symptom and inflammation and achieve remission. 
However, the effectiveness of treatment was severely limited by poor adherence to medication. Adherence to medication in RA patients is not optimal, with only approximately $66 \%$ of RA patients taking medications as prescribed. Thus, interventions to improve medication adherence for patients with RA are needed. The study explored the effects of electronic health interventions on medication adherence and disease activity in patients with rheumatoid arthritis.

Condition being studied: Rheumatoid arthritis.

\section{METHODS}

Search strategy: The key search terms were "e-health OR $\mathrm{m}$-health OR telehealth OR remote OR internet OR website OR computer OR network OR online OR media OR video OR message OR e-mail OR radio OR tablet OR handheld device OR personal digital assistant OR phone OR telephone OR smartphone OR mobile phone OR cell phone OR portable data terminal OR podcast OR application OR software" AND "rheumatoid arthritis OR arthritis, rheumatoid" AND "randomized controlled trial OR controlled clinical trial OR random*".

Participant or population: Inclusion criteria for patients were: (a) confirmed 2010 American College of Rheumatology criteria (ACR)/European League Against Rheumatism (EULAR) classification criteria for diagnosis of RA; (b) aged 18 years or older. Excluded criteria for patients were: (a) hearing impairment; (b) severe cognitive or mental disorder; (c) participation in other educational programs.

Intervention: The inclusion criteria were: For e-Health, based on the recommendations, we mainly focused on two categories: web-Health via desktop web access (traditional Internet-based), and $\mathrm{m}$-Health (e.g., APP-based). Telehealth, defined as the remote delivery of health care by telecommunication tools, including telephones and the internet, enables accessible and appropriate care and has provided opportunities of communication with patients, support, and monitoring The excluded criteria were: lack of telephone or lack of Internet.

Comparator: The inclusion criteria were: Waiting-list control, usual care, or active controls (e.g., health education). Patients in the control group only received standard care from the nursing staff, which consisted of discharge instructions. Discharge instructions included instructions about medication and basic health advice appropriate for RA patients. The excluded criteria were: participation in other educational programs.

Study designs to be included: Randomized controlled trials will be included; Otherwise, they will be excluded.

Eligibility criteria: The inclusion criteria were: (a) types of studies: randomized controlled trials published as full text; (b) types of participants: (b) confirmed 2010 American College of Rheumatology criteria (ACR)/European League Against Rheumatism (EULAR) classification criteria for diagnosis of RA; aged 18 years or older.; (c) types of interventions: studies comparing the effectiveness of e-health and non-e-health-based controls. E-healthbased health care was defined as a medical practice delivered via smartphone (e.g., app, mobile website, e-mail), telephone (e.g., SMS, teleconferencing), computer (e.g., website, app), or other electronic equipment.; (d) types of outcome measures: studies assessing disease activity, medication compliance and quality of life with scales, but not a single item. The exclusion criteria were: (a) hearing impairment; (b) severe cognitive or mental disorder; (c) participation in other educational programs.

Information sources: We researched PubMed, Cumulative Index Nursing and Allied Health Literature, Cochrane Central Register of Controlled Trials, Web of Science and Embase up to December 2020. In addition, we also conducted manual searches of potentially eligible references. 
Main outcome(s): In this study, medication compliance, quality of life and disease activity were focused on as the primary outcomes of interest. Studies were included only if they specified medication compliance, quality of life and disease activity as the primary outcomes; otherwise, they would be excluded. We assessed participants' disease activity by erythrocyte sedimentation rate (ESR), Creactive protein (CRP) and Disease Activity Score 28 (DAS28). Medication adherence was measured by Compliance Questionnaire Rheumatology. studies assessing disease activity, medication compliance and quality of life with scales, but not a single item.

\section{Additional outcome(s): None.}

Data management: Review Manager 5.3 was used for data analysis. If statistical heterogeneity existed, the effect size of the subgroups was determined based on the different characteristics of the studies to explore the source of heterogeneity. The sensitivity of the results was analyzed using STATA 15.0 by comparing the effect sizes and confidence intervals (Cls) of the remaining studies after eliminating each study at a time. Two reviewers independently assessed the risk of bias for each included article using the assessment tool from the Cochrane handbook (version 5.1.0). Disagreements were resolved by discussion or, if necessary, on the advice of a third reviewer. Two reviewers independently extracted the following data: authors, year of publication, countries, sample size, interventions (content, delivery, duration, time of each activity and frequency), control conditions, outcomes, measured time points, mean and standard deviation).

Quality assessment / Risk of bias analysis: Selection bias, performance bias, detection bias, attrition bias, reporting bias and other bias were assessed using the following components: random sequence generation, allocation concealment, blinding of participants, blinding of personnel, blinding of assessment of outcomes, incomplete outcome data, and selective outcome reporting. Review Manager 5.3 was used to present the conclusion of the risk of bias assessment. The quality of evidence was categorized by the GRADE methodology as four grades: high, moderate, low and very low. Risk of bias, inconsistency, indirectness, imprecision, and publication bias were measured with the handbook in GRADE profiler 3.6 software.

Strategy of data synthesis: A random-effect model was chosen to calculate the pooled results for different characteristics of the studies. For continuous outcomes, the standard mean difference (SMD) was used since the same outcome across separate studies was measured by different instruments. Cohen's categories were used to calculate the overall effect size as follows: $0.2-0.5=$ small effect size, $0.5-0.8$ $=$ moderate effect size, $>0.8=$ large effect size. Statistical heterogeneity among the studies was examined quantitatively by Cochran's $Q$ test and the $I^{2}$ statistic, visually by forest plots. Heterogeneity was rated as follows: $\mathbf{0} \% \mathbf{- 4 0} \%$, not important; $30 \%-60 \%$, low heterogeneity; $50 \%-90 \%$, moderate heterogeneity; and $75 \%-100 \%$, high heterogeneity. If statistical heterogeneity existed, the effect size of the subgroups was determined based on the different characteristics of the studies to explore the source of heterogeneity. The sensitivity of the results was analyzed using STATA 15.0 by comparing the effect sizes and confidence intervals (Cls) of the remaining studies after eliminating each study at a time. Selective reporting outcomes bias of each study was assessed by comparing the protocol with the corresponding published article. Meanwhile, according to the recommendations in the Cochrane Handbook, publication bias was evaluated because fewer than 10 studies were included.

Subgroup analysis: We will consider subgroups such as disease activity, medication compliance and quality of life.

Sensibility analysis: Statistical heterogeneity among the studies was examined quantitatively by Cochran's $Q$ 
test and the $I^{2}$ statistic, visually by forest plots. Heterogeneity was rated as follows: 0\%-40\%, not important; $30 \%-60 \%$, low heterogeneity; $50 \%-90 \%$, moderate heterogeneity; and $75 \%-100 \%$, high heterogeneity. If statistical heterogeneity existed, the effect size of the subgroups was determined based on the different characteristics of the studies to explore the source of heterogeneity. The sensitivity of the results was analyzed using STATA 15.0 by comparing the effect sizes and confidence intervals (Cls) of the remaining studies after eliminating each study at a time.

\section{Language: English.}

\section{Country(ies) involved: China.}

Keywords: meta-Analysis, electronic health interventions, medication compliance, disease activity, rheumatoid arthritis.

Dissemination plans: I intend to publish the review on completion.

Contributions of each author:

Author 1 - Jing Wang - WJ contributed to the acquisition and analysis of data. The manuscript was drafted by WJ.

Email: jingwang0513@163.com

Author 2 - Pingyu yang - The author provided statistical expertise and contributed to the development of the selection criteria, and the risk of bias assessment strategy.

Email: 542017195@qq.com

Author 3 - Huling Cao - The author read, provided feedback and approved the final manuscript.

Email: 1463178375@qq.com

Author 4 - Jielin Ben - The author contributed to the development of the selection criteria, and the risk of bias assessment strategy.

Email: benjl121212@163.com

Author 5 - Yingying Gao - The author read, provided feedback and approved the final manuscript.

Email: gaoyy123123@163.com

Author 6 - Xueqin Wang - The author contributed to the development of the selection criteria, and the risk of bias assessment strategy.

Email: 1463178275@qq.com

Author 7 - Weiqun Weng - The author read, provided feedback and approved the final manuscript.

Email: weng1463178375@163.com 